\title{
TRIM: An architecture for transparent IMS-based mobility
}

\author{
Ivan Vidal ${ }^{\mathrm{a}, *}$, Antonio de la Oliva ${ }^{\mathrm{a}}$, Jaime Garcia-Reinoso ${ }^{\mathrm{a}}$, Ignacio Soto ${ }^{\mathrm{b}}$ \\ a Departamento de Ingeniería Telemática, Universidad Carlos III de Madrid, Avda. de la Universidad 30, 28911 Leganés, Madrid, Spain \\ ${ }^{\mathrm{b}}$ Departamento de Ingeniería de Sistemas Telemáticos, Universidad Politécnica de Madrid, Avada. Complutense 30, 28040 Madrid, Spain
}

Keywords:

SIP

IMS

Transparent mobility

Handover management

\section{A B S T R A C T}

In recent years, the development and deployment of new wired and wireless access net work technologies have made the ubiquitous Internet a reality. Users can access anywhere and anytime to the broad set of value added Internet services, which are delivered by means of the IP protocol. In this context, 3GPP is currently developing the IP Multimedia Subsystem (IMS), as a key element that allows to evolve from the ubiquitous access to the Internet services towards a next generation network model, by providing a set of essen tial facilities such as session control, QoS, charging and service integration. Nevertheless, several open issues still need consideration before the future Internet becomes real, such as supporting user mobility in IP networks. Although mobility support in the Internet is receiving much attention, IMS networks present inherent particularities that require fur ther analysis. The solutions proposed so far for IMS do not support mobility transparently to the end user applications, or address the problem by introducing complex changes to the IMS infrastructure. This paper presents TRIM, an architecture for transparent IMS based mobility. TRIM supports mobility in IMS networks transparently to the end user applications, which are unaware of the handover management procedures executed between the mobile node and the network. We have performed several experiments with a TRIM prototype, using a real IMS testbed with 3G and WLAN access networks, validating the proposal for UDP and TCP based applications.

\section{Introduction}

IP is becoming the cornerstone technology for commu nication networks. In this context, telecommunication operators are pushing the introduction of the IP Multime dia Subsystem (IMS) in their IP networks to provide access and session control. IMS enables in IP networks services traditionally associated with circuit switched networks such as telephony. These services can combine several media and QoS requirements. IMS was developed by $3 \mathrm{GPP}^{1}$ for UMTS networks, and it is currently working

\footnotetext{
* Corresponding author.

E-mail addresses: ividal@it.uc3m.es (I. Vidal), aoliva@it.uc3m.es (A. de la Oliva), jgr@it.uc3m.es (J. Garcia-Reinoso), isoto@dit.upm.es (I. Soto).

${ }^{1}$ http://www.3gpp.org.
}

together with ETSI TISPAN to extend the IMS specification for any type of access technology.

Mobility and the ability to access the Internet every where and anytime are characteristics that users have come to expect. Mobility support in IP networks has been studied for some time. The key issue is that, in IP, addresses are used both as identifier and locator. So when a node moves, from the point of view of the applications we need to maintain its IP address (because it is an identifier) but from the point of view of the IP layer we should change the IP address to one topologically correct. The IETF$^{2}$ has standardized the Mobile IP (MIP) protocols both for IPv4 [1] and for IPv6 [2] to provide mobility support in IP net works. Mobile IP makes mobility transparent to any commu nication layer above IP, including the applications and,

\footnotetext{
${ }^{2}$ http://www.ietf.org.
} 
therefore, a node is able to change the IP subnetwork it is using to access the Internet without losing its active com munications. Note that some networks support the move ment of nodes without requiring them to change their IP address. In these networks mobility is transparent to the nodes at the IP layer, so layers above IP are not affected by mobility and no additional mobility support is required. For example, this is the case when a mobile node (MN) moves within the same UMTS network. Nevertheless, there are trends that will make mobility among different net works, meaning the change of IP address and consequently the need for a mobility support mechanism that deals with this change, much more common. Examples of these trends are the use of devices with several technologies that connect through the most appropriate one in each situation, the availability of new access technologies that will co exist to cover different requirements, and operators that integrate several offerings (fixed and mobile access).

Mobile IP is a good solution to provide mobility support but its integration in IMS is far from trivial [3 5]. This is essentially because MIP hides from the application layer, including the IMS control, the IP address used by a MN in an access network, but IMS requires this address to reserve resources in the access network for the traffic of the ser vices used in the node.

Another alternative is to use the Session Initiation Pro tocol (SIP) to support mobility [6] in IP networks. In this re spect, 3GPP has proposed a set of mechanisms to maintain service continuity in the event of terminal mobility [7]. Using SIP to handle mobility presents the advantage of not requiring additional mechanisms outside the signaling used in IMS. But the traditional SIP mobility support is not transparent to the application layer. This means that appli cations have to be programmed with mobility in mind which is inconvenient. Section 5 reviews different ap proaches to provide mobility support in IP networks with IMS, comparing them with this work.

This paper proposes TRIM (described in Section 3), an architecture providing mobility support in IMS based net works. It is based on SIP signaling and does not require any modifications to the IMS specifications, which is an impor tant advantage over the alternative of using Mobile IP in an IMS network. TRIM makes the change of IP address in a mobile node, required to support the movement, transpar ent to the applications running in the mobile node and also to the peer nodes. We implemented a prototype of the TRIM architecture and tested the proposal using different access networks (3G and IEEE 802.11) and both UDP and TCP user traffic (see Section 4).

\section{Background on IMS}

The IP Multimedia Subsystem (IMS) is an architectural framework introduced by 3GPP as part of the standardiza tion process of the UMTS technology. It was designed as a key element to enable the delivery of value added mul timedia services, including those that were traditionally provided through circuit switched networks, over packet switched networks. In this respect, the IMS is designed to support facilities related to session control, QoS, charging, interworking with the Internet and circuit switched net works, service control and creation, integration of heteroge neous access technologies, security and roaming. Fig. 1 depicts a simplified overview of the architecture defined by 3GPP for the IMS (see [8] for further details).

In the IMS architecture, session control is based on the Session Initiation Protocol (SIP) and the Session Descrip tion Protocol (SDP). Although these are Internet protocols standardized by the IETF (see $[9,10]$, respectively), session control in the IMS follows a specific profile for SIP and SDP defined by the 3GPP [11]. In the figure, the Call Session Control Functions (CSCFs) are the functional entities responsible of processing the SIP signaling messages: the Proxy CSCF (P CSCF) is the first contact point between the UE and the IMS, and processes every SIP message that originates or terminates in the UE; the Interrogating CSCF (I CSCF) is the contact point in the user home network for every incoming session addressed to the UE; the Serving CSCF (S CSCF) performs session control and registration functionalities. The S CSCF may also route incoming SIP signaling messages to a set of Application Servers (ASs), which provide services to the end user. In addition, other functional entities are specially relevant in the architec tural framework: the user databases, i.e. the Home Sub scriber Server (HSS), the Subscriber Location functions (SLF), and the Policy Control and Charging Rules Function (PCRF), which provides policy control decision and flow based charging control functionalities.

\section{The TRIM architecture}

This section describes the TRIM architecture. TRIM sup ports mobility transparently to the end user applications, which can use UDP or TCP in the user plane, and are una ware of the handover management procedures executed between the MN and the network. Unlike the previous pro posals, the described solution does not require changes to the IMS infrastructure as defined by 3GPP [8]. In addition, the proposal does not entail any upgrades to the corre spondent node $(\mathrm{CN}$, the node communicating with the $\mathrm{MN}$ ) architecture, being the extensions only necessary in the $\mathrm{MN}$ and the home network corresponding to the TRIM user. Fig. 2 depicts a general overview of the TRIM archi tecture, highlighting the relation of its different compo nents with the IMS infrastructure. ${ }^{3}$

The main component of the TRIM architecture is a SIP Application Server (AS), namely the TRIM AS. Each user sub scribed to the transparent handover service described in this section will be served by a TRIM AS located in its home network. This Application Server will receive all the SIP sig naling messages corresponding to the multimedia sessions originated or terminated in the MN. This way, the TRIM AS stays on the signaling path utilized between the MN and any $\mathrm{CN}$. To guarantee scalability and redundancy, the home network may contain a certain number of TRIM ASs.

\footnotetext{
${ }^{3}$ In this figure, it is assumed that the MN has acquired connectivity from a visited network and uses a P-CSCF located in that network. However, a mobility scenario is possible where the MN uses a P-CSCF located in the home network of the mobile user. The procedures presented along this section can handle transparent mobility in both scenarios.
} 
UE: User Equipment

P-CSCF: Proxy-Call Session Control Function S-CSCF: Serving-Call Session Control Function I-CSCF: Interrogating-Call Session Control Function
PCRF: Policy and Charging Rules Function HSS: Home Subscriber Server SLF: Subscriber Location Function

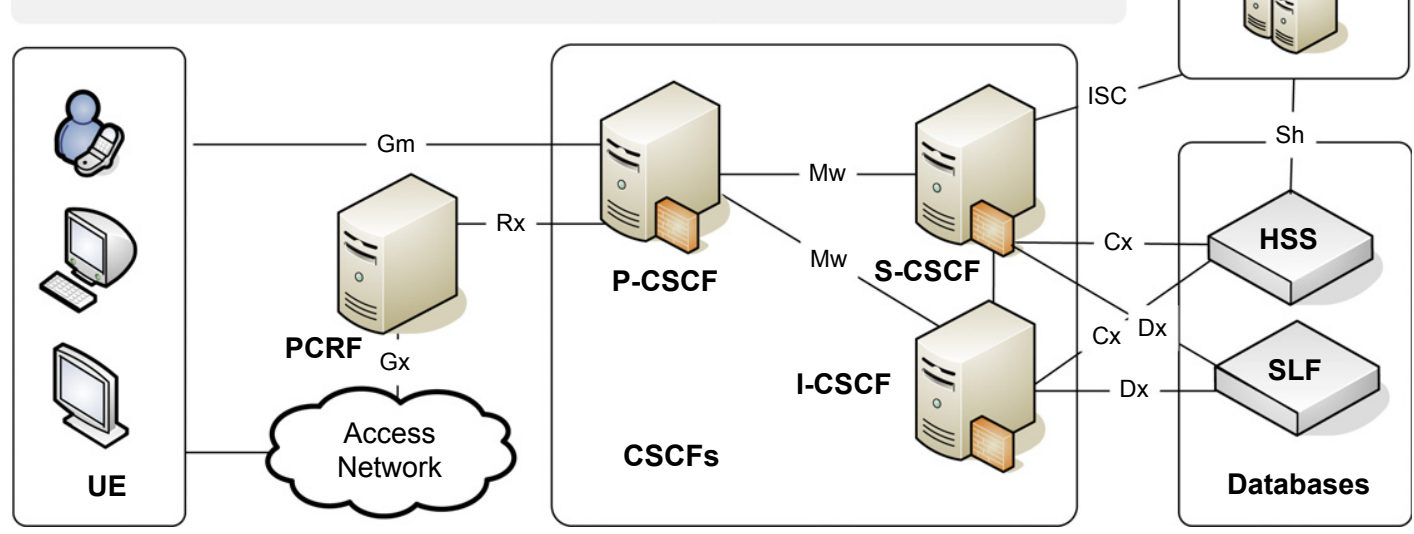

Fig. 1. IMS architecture.

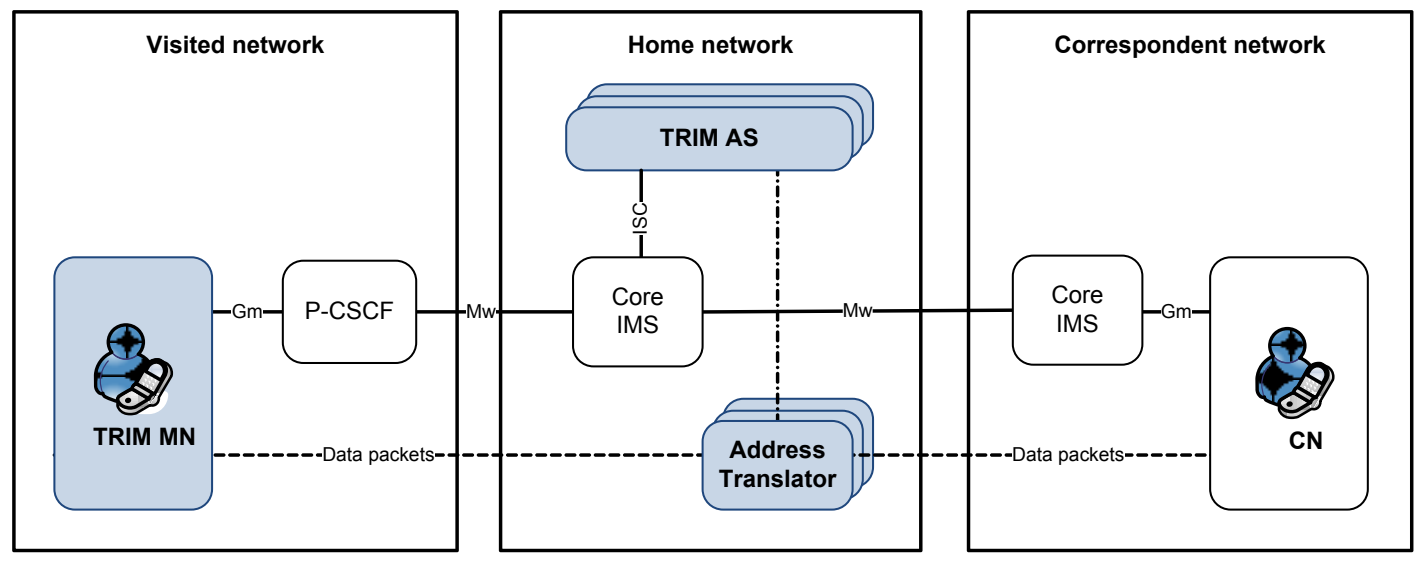

Fig. 2. Overview of the TRIM architecture.

On the other hand, the TRIM AS controls a set of address translators, which are located in the home network of the mobile user. ${ }^{4}$ As the AS stays on the signaling path between the $\mathrm{MN}$ and the $\mathrm{CN}$, it ensures that all the media exchanged by them in the user plane passes through one of these translators. The address translator is configured by the TRIM AS to address the media received from the $\mathrm{CN}$ to the appropriate location where the $\mathrm{MN}$ is willing to re ceive it. Analogously, it forwards the media received in the opposite direction, i.e. from the $\mathrm{MN}$ to the $\mathrm{CN}$. In concrete, the address translator simply changes the IP addresses and ports contained within the data packets according to the configuration provided by the TRIM AS. Therefore, the $\mathrm{CN}$ always observes the same remote addressing information for the MN, no matter where the latter is located. Again, for the sake of scalability and redundancy, there may be

\footnotetext{
${ }^{4}$ Note that the TRIM AS and the address translators can be deployed in different network nodes. In this case, a protocol such as DIAMETER is needed to communicate them (a similar use case is described in [12]).
}

a certain number of address translators within the home network.

As it will be subsequently explained, the TRIM enabled MN keeps the AS updated with its current addressing information. This information, as well as the mechanisms to update it in the TRIM AS, are transparent to the end user applications that run on the MN. The AS configures and up dates the address translator assigned to the $\mathrm{MN}$ with the current addressing information that it utilizes in the user plane. Thus, the MN can always communicate with the $\mathrm{CN}$ irrespective of its location.

Hence, the presented solution introduces two new intermediate elements, the TRIM AS and the address trans lator, as well as the architecture of a TRIM enabled MN. In the user plane, the address translator plays a similar role to the home agent (HA) in MIP. Nevertheless, the proposal does not require changes to the IMS infrastructure as de fined by 3GPP. Next subsections cover (1) the details of the TRIM architecture at the MN, as well as the signaling procedures that are necessary (2) to establish a multimedia 
session between the $\mathrm{MN}$ and the $\mathrm{CN}$ and (3) to handle the transparent handover of the MN in IMS networks.

\subsection{Architecture of a TRIM enabled MN}

As previously indicated, TRIM guarantees that the addressing information corresponding to the current loca tion of the $\mathrm{MN}$, as well as the procedures to notify this information to the TRIM AS, are transparent to the end user applications that run on the mobile device. To make this possible, we define new functionalities to an IMS enabled terminal (i.e. the $\mathrm{MN}$ ).

Fig. 3(a) illustrates the reference model that has been considered in this article for an IMS enabled terminal. In this model, an IMS SIP User Agent (IMS SIP UA) is the func tional entity in the terminal in charge of executing all the SIP signaling procedures with the IMS network (e.g. regis tration, session establishment and release, etc.). The SIP UA can be triggered when some relevant lower layer event takes place, for instance when the terminal gets IP connec tivity to a new access network and the address of a new $P$ CSCF has been obtained. These lower layer triggers can imply certain signaling procedures to be executed (e.g. an IMS registration after obtaining IP connectivity to a new access network). The SIP UA is enabled to open network sockets towards the lower layers, to support the exchange of SIP signaling messages with the IMS network.

On the other hand, the user terminal may execute a set of applications requiring network connectivity, such as VoIP, online gaming or IPTV. These applications can contact the SIP UA in order to establish multimedia sessions to wards remote parties. For instance, in case of a VoIP call, an audio session needs to be established with the callee be fore any voice data can be sent or received. In this case, the VoIP application running on the terminal would generate a description of the audio session to be established, accord ing to the procedures specified in the 3GPP profile of SDP and would request the SIP UA to establish the session with the callee. In addition, applications are allowed to open network sockets in order to support the data exchange that is necessary for an appropriate operation (e.g. to send and receive audio packets in case of VoIP).
Nevertheless, under this model, mobility is not trans parent to applications. In fact, when the MN gains IP con nectivity to a new access network:

- Any ongoing multimedia session, established with a CN, needs to be re established, in order to guarantee an appropriate resource reservation in the new access net work and to inform the $\mathrm{CN}$ of the new addressing infor mation that must be used in the user plane. In this process, the application must provide the SIP UA with a new SDP description, reflecting the new addressing information for the media components that will be exchanged within the session.

- Any network socket bound to an IP address that belongs to the old network becomes invalid. Hence, applications need to re configure or open new network sockets.

Fig. 3(b) shows the extensions proposed in this paper to an IMS enabled MN to support transparent handover for the end user applications. In this new architecture, any call to the lower layers to retrieve the MN's IP address receives back a private/loopback IP address. In addition, TRIM intro duces two new functional entities into an IMS terminal: a handover manager and an address translator.

The handover manager ( $H M)$ is located in the user space of the MN, and acts as an intermediary between the appli cations and the SIP UA. This entity receives requests from the applications to establish, modify and release multime dia sessions towards any specific destination. In addition, each request can contain an SDP payload. If so, the payload comprises the description of the different media compo nents (e.g. audio or video) that the application wants to in clude in the multimedia session. For each media, among other things, the application indicates the addressing infor mation (IP address and port) where it is willing to receive the component.

The HM modifies the SDP payload received from the application, changing the addressing information associ ated with each media component. In particular, the man ager replaces the internal IP address, which is visible for the applications, by the real IP address that the MN got from the current access network (port numbers are left

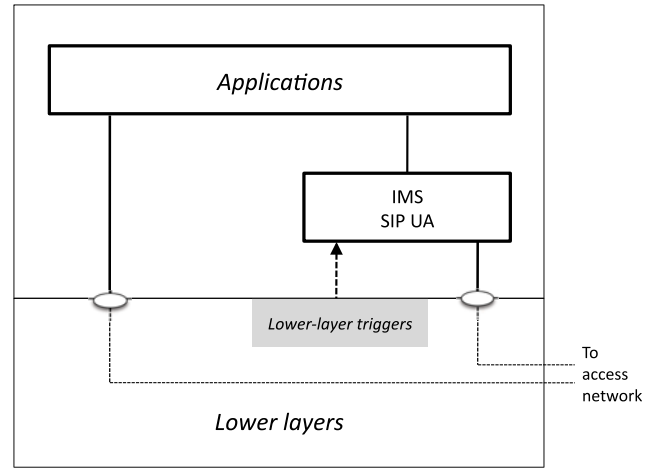

(a) Architecture of an IMS terminal

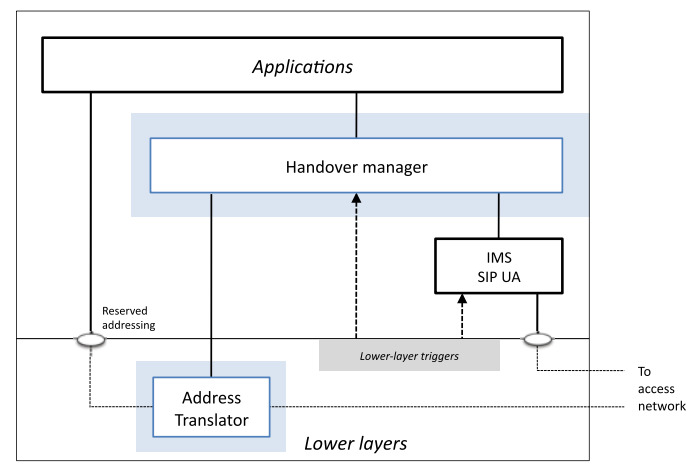

(b) Architecture of a TRIM enabled MN

Fig. 3. Introducing the TRIM architecture into an IMS terminal. 
unchanged). Then, the handover manager forwards the request to the SIP UA, which performs the signaling pro cedures that are necessary to serve the application re quest. Additionally, the manager stores the current SDP payload and an identifier of the multimedia session for further use.

Analogously, the SIP UA can receive a request from a re mote party to establish, modify or release a multimedia session with any application that runs on the MN. This request is sent to the handover manager. As before, the request can contain an SDP payload. This payload indi cates, for each described media component, the addressing information where the remote party wants to receive the component. This addressing information does not need to be changed and, consequently, the request is transparently forwarded to the intended application.

The handover manager will be triggered by the lower layers when the MN gets IP connectivity to a new access network. If this happens, the HM will retrieve the infor mation of the ongoing multimedia sessions. For each of them, it updates the stored SDP payload, changing the addressing information for each media component. In concrete, the manager replaces the old IP address with the IP address that the MN has obtained from the new ac cess network. Then, the handover manager contacts the SIP UA to modify the multimedia session. As a result, a signaling procedure is executed between the SIP UA and the TRIM AS, and the AS obtains the new SDP description for the multimedia session from the MN. With this infor mation, the TRIM AS can update the address translator configuration, so that subsequent data packets, corre sponding to the multimedia session, are properly routed towards the new location of the MN. The details of this signaling procedure are provided in Section 3.3. The pro cedure is executed for each multimedia session estab lished with the MN.

The address translator is located in the kernel space within the MN. This address translator runs locally at the $\mathrm{MN}$ and is independent from the set of address translators shown in Fig. 2. As previously indicated, the address trans lators illustrated in that figure are located in the home net work, and are responsible of (1) addressing the data traffic received from the $\mathrm{CN}$ to the appropriate location of the MN and (2) providing the $\mathrm{CN}$ with stable remote addressing information for the MN. As we explain next, the address translator located at the MN complements this functional ity, allowing applications that run locally at the MN to uti lize internal addressing information that remains stable independently of the MN location.

In general, an application can open a set of network sockets to exchange traffic within a multimedia session. Each of these sockets is bound to the internal IP address and to a given port. Therefore, traffic sent through these sockets includes the internal address as the source IP ad dress. The address translator behaves like a NAT, chang ing the source IP address of every packet sent in the uplink direction (from applications to network) by the real IP address assigned to the MN in the current access network.

On the other hand, as previously explained, the remote party in a session receives an SDP payload with the real addressing information corresponding to the MN. There fore, packets belonging to the session will be addressed from the remote party to the current IP address of the de vice. The address translator also replaces the destination IP address of every incoming packet addressed to the applica tions running in the MN, with the internal IP address.

This way, applications interact with the HM to estab lish, modify or release multimedia sessions. The HM prox ies requests between applications and the SIP UA changing the local addressing information in the SDP pay loads. This way, the handover manager hides the current addressing information to the applications, that always observe an invariant IP address (the internal IP address), no matter where the MN is located. On the other hand the HM always provides the SIP UA with appropriate SDP payloads, containing the current addressing informa tion corresponding to the $\mathrm{MN}$. In the user plane, the ad dress translator at the $\mathrm{MN}$ changes the addressing information of the data packets, so that local applications always receive data in the sockets they are bound to, while the remote parties receive traffic originated from the real address of the MN.

\subsection{Procedures for session establishment}

Whenever a local application, running on the MN, needs to exchange media with an application running on a $\mathrm{CN}$, the MN must first establish a multimedia session with the $\mathrm{CN}$. This procedure is triggered by the local application, which generates an SDP payload describing the session and requests the HM to establish a multimedia session with the $\mathrm{CN}$. As we have explained, the HM updates the local addressing information contained in the SDP payload, and proxies the request to the SIP UA, which is in charge of the session setup. The signaling process corresponding to the session establishment is illustrated in Fig. 4. It is as sumed, in this figure, that the MN establishes the multime dia session from a visited network where it needs to reserve local resources.

The signaling process is initiated by the SIP UA in the MN, by sending a SIP INVITE request that, according to the IMS routing mechanisms, arrives to the S CSCF allo cated to the user in the home network. This request con tains an SDP offer, which is the SDP payload provided by the HM. If the user has contracted the transparent mobility service described in this paper, then the IMS initial filter criteria will indicate that the request should be routed to a TRIM AS, also located in the home network.

The TRIM AS is a SIP Back to Back User Agent (B2BUA), as defined in [9]. Assuming this role, it stays on the path of subsequent SIP requests and responses exchanged be tween the MN and the $\mathrm{CN}$. In addition, the TRIM AS pro cesses the SDP offer received from the MN. In concrete, for each media component included in the offer:

- The IP address and port included in the offer represent the addressing information where the MN is willing to receive the data traffic of the media component. The TRIM AS selects an address translator from the home network (see Fig. 2), and requests from the translator a new binding for the IP address and port. As a result, 


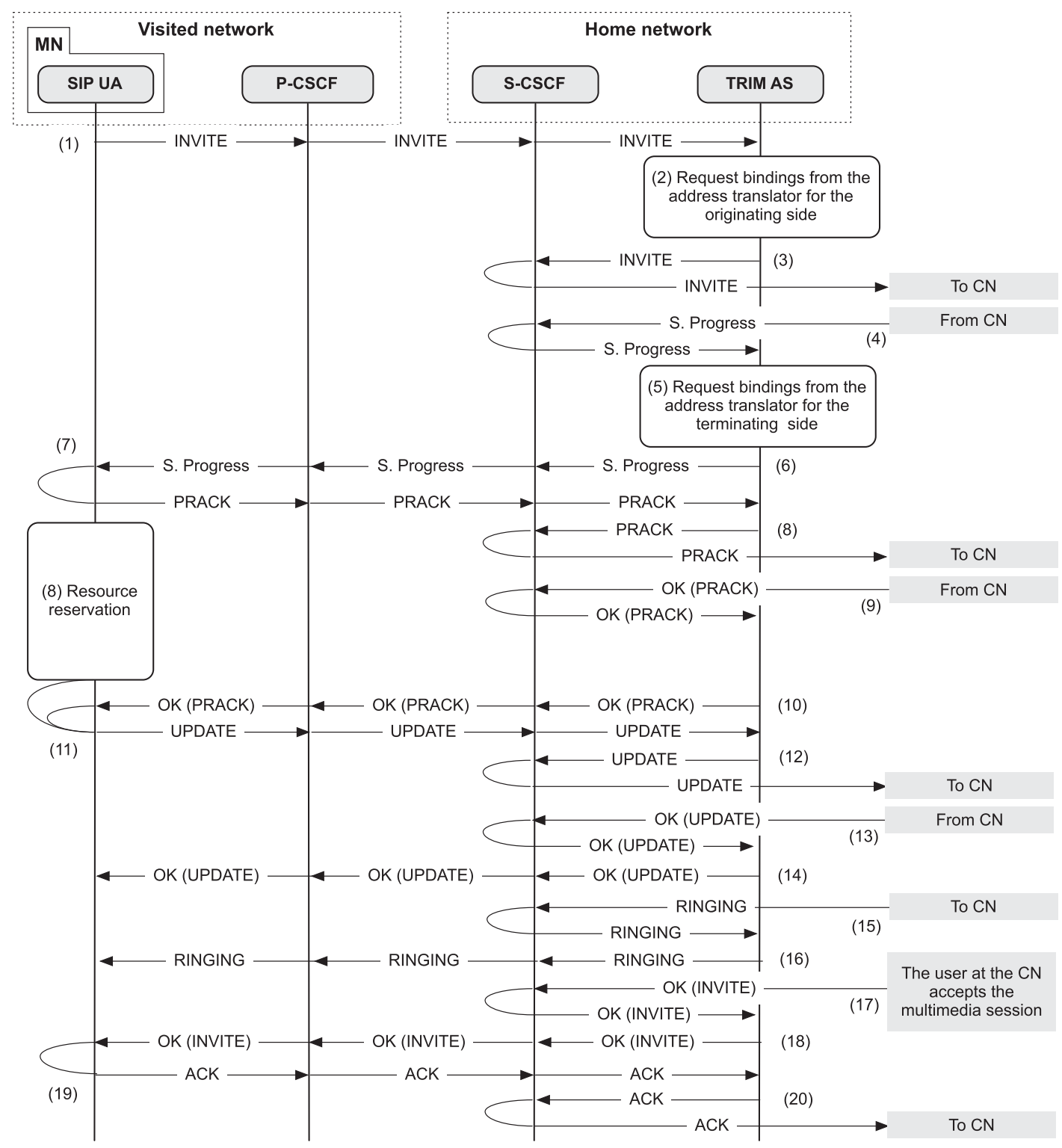

Fig. 4. IMS signaling for session establishment.

the address translator returns a new pair of IP address and port, which should be used by the $\mathrm{CN}$ to send the data traffic corresponding to the media component. ${ }^{5}$

- The TRIM AS replaces the IP address and port specified for the media component with the binding obtained from the address translator.

Afterwards, the TRIM AS generates a new INVITE request, containing the modified SDP offer, and sends this request to

\footnotetext{
${ }^{5}$ For the sake of scalability, we assume that there may be several address translators in the home network. In addition, each address translator may be assigned a set of IP addresses. This should enable the TRIM AS to successfully obtain a binding for the IP address and port corresponding to the media component.
}

wards the $\mathrm{CN}$. As this request is related with the INVITE re quest previously received at the AS, the new request is routed back to the S CSCF. Finally, after processing the re quest, the S CSCF forwards it towards the core IMS of the CN.

Eventually, the $\mathrm{CN}$ receives the INVITE request and an swers it back with a SIP Session in Progress response. This response contains an SDP answer, describing the media components accepted by the destination. At some point, the Session in Progress response reaches the TRIM AS, that processes the SDP answer. For each media component in cluded in the answer:

- The IP address and port included in the answer indicate the addressing information where the $\mathrm{CN}$ is willing to accept the data traffic corresponding to the media 
component. Again, the TRIM AS requests from the address translator, which had been previously selected, a binding for the IP address and port. As a result, the address translator returns a pair of IP address and port, which should be used by the MN as the destina tion of the data traffic corresponding to the media component.

- The TRIM AS replaces the IP address and port specified for the media component with the binding obtained from the address translator.

Finally, the TRIM AS generates a new Session in Progress response to the initial INVITE request that was received from the MN. In this response, the AS includes the modified SDP answer. The Session in Progress response is routed back to the MN, passing through the S CSCF and the P CSCF. At the MN, the SIP UA notifies the HM about the ses sion disposition, providing it with the SDP answer. As it has been previously indicated, the HM proxies the request to the application that triggered the session setup. Therefore, the application is notified about the addressing informa tion that it should use to send the data traffic within the multimedia session towards the $\mathrm{CN}$.

The session establishment continues according to the regular IMS procedures. When the MN receives the SIP OK response to the INVITE request, the session has been established. At this point, the application is notified through the HM and the data exchange can start in the user plane. Fig. 5 illustrates the address translation proce dures that take place in the user plane after the session establishment.

The case where the $\mathrm{CN}$ initiates the session establish ment towards the MN follows a similar procedure. Beyond the direction of the SIP signaling flows (in this scenario the INVITE request is received at the MN), the procedures exe cuted by the TRIM AS remain basically unchanged.

\subsection{Handling the transparent handover}

When the MN moves to a new network and obtains IP connectivity from the new location, the SIP UA and the handover manager will be triggered by the lower layers. At this point, the SIP UA can register the new contact URI in the S CSCF, where the MN is now reachable, and de register the previous URI. On the other hand, the handover manager must ensure that the TRIM AS is informed about the new addressing information that must be used for every multimedia session that involves the MN. In this respect, as it has been previously explained, the manager retrieves the information about the ongoing multimedia sessions and, for each session, it updates the corresponding SDP payload, changing the addressing information for each media component to reflect the new IP address where the mobile node will receive the media. Next, the manager re quests from the SIP UA the modification of the ongoing sessions, according to the new SDP parameters. The modi fication of an existing multimedia session is initiated by the SIP UA, by sending an INVITE request (a re INVITE if the $P$ CSCF has not changed or a new INVITE request with a Replaces header in case that the P CSCF has changed). Fig. 6 depicts the signaling procedure executed between the SIP UA and the TRIM AS for each ongoing session, under the assumptions that the MN needs to use a P CSCF and re serve local resources within the new access network.

In this signaling procedure, the TRIM AS receives in the INVITE request the updated information about the multi media session that is being modified. This information is in cluded in the SDP offer carried by the request. In concrete, the SDP offer specifies, for each media component, the new IP address and port where the $\mathrm{MN}$ is willing to receive the data traffic associated with the component. The TRIM AS stores this updated information and answers back the request with a Session in Progress response, that includes

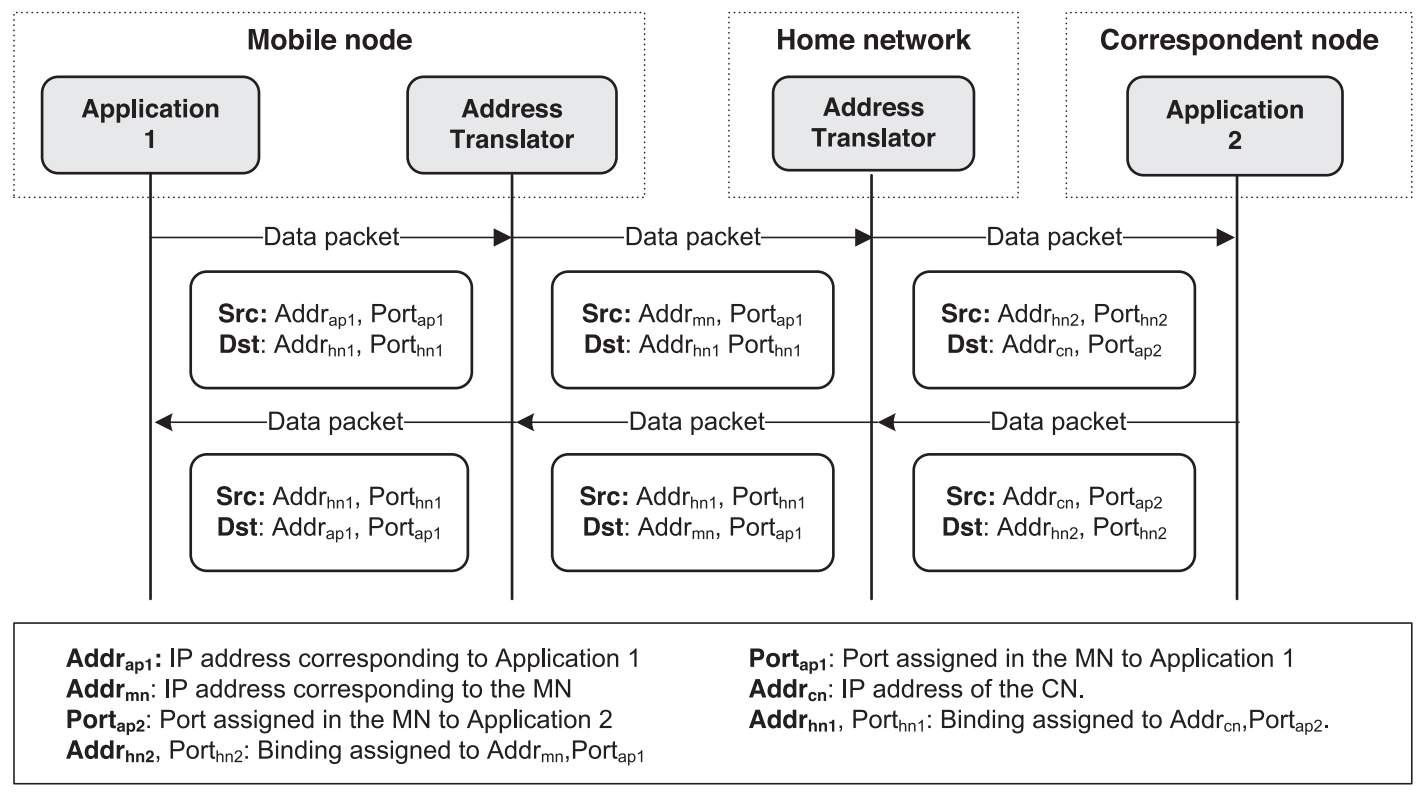

Fig. 5. Management of data packets in the user plane. 


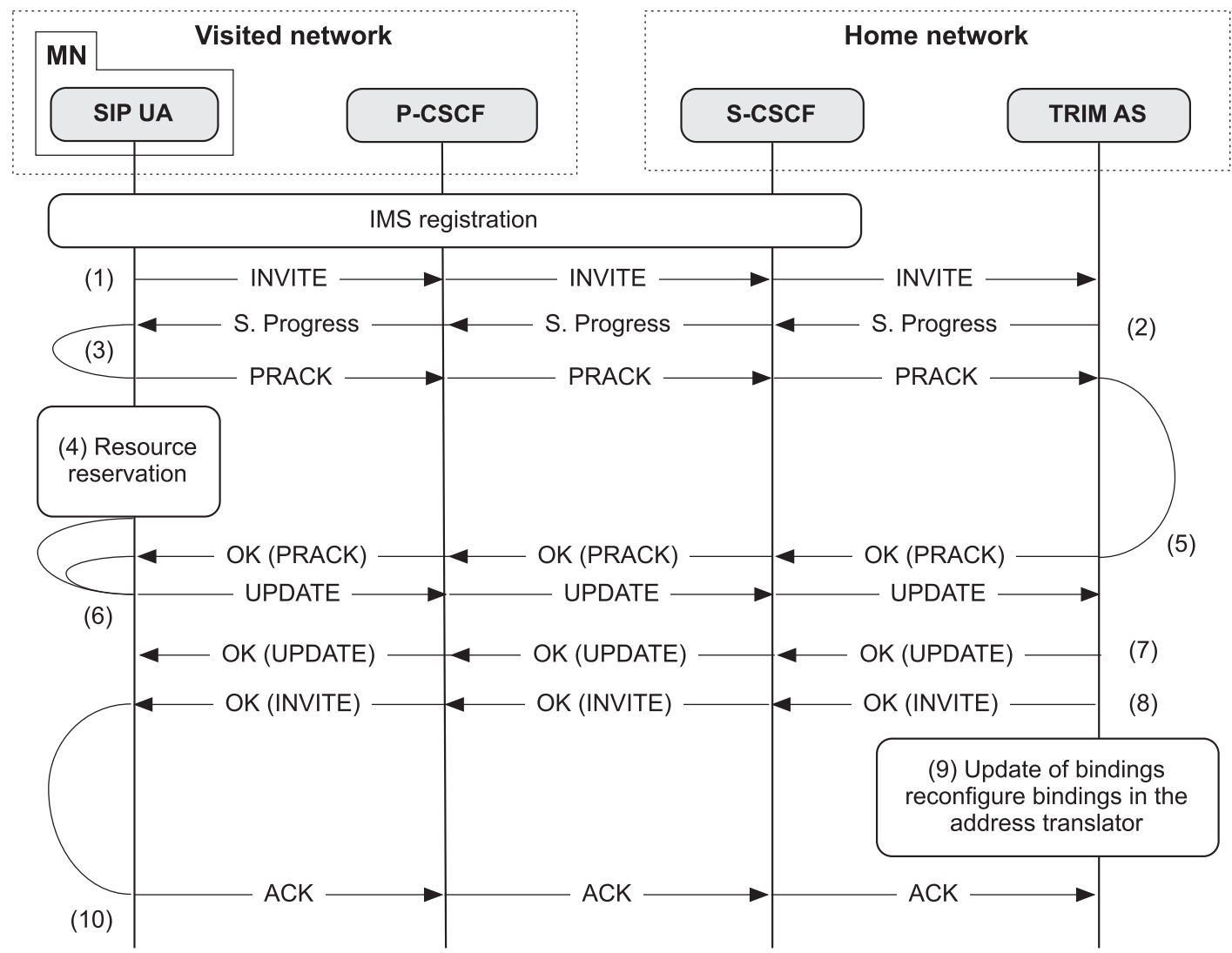

Fig. 6. IMS signaling for handover management.

an SDP answer. This SDP answer does not contain any changes to the addressing information that must be used by the MN to send data traffic (i.e. the MN should continue sending data within the multimedia session towards the address translator). When the SIP UPDATE request arrives to the TRIM AS, meaning that the MN has successfully accomplished the local resource reservation, the TRIM AS accepts the session modification by sending a SIP OK re sponse to the INVITE request, and contacts the address translator in order to update the addressing information corresponding to each media component within the multi media session. Consequently, the address translator starts forwarding the media belonging to the session to the new location of the mobile node. In addition, when the SIP UA receives the $\mathrm{OK}$ response, the $\mathrm{HM}$ is notified about the suc cessful session modification, and configures the new IP ad dress assigned to the $\mathrm{MN}$ in the local address translator. Additionally, the HM configures the lower layers to use the new interface for the uplink traffic.

The proposal described in this section is valid in a hard handover and in a soft handover scenario. The difference between both cases lies in the instant when local resources are released in the old access network. While in hard hand over resources are released before gaining connectivity to the new access network, in soft handover this resources are be retained until data traffic is received through the new access network, being released afterwards.

\section{Evaluation of the proposal}

This section presents the results of the validation of the mobility solution described in the previous section. In or der to do so, a complete prototype of the solution was implemented and different handovers for TCP and UDP transport protocols were performed. In the following sub sections we provide insights of the behavior of the proto type implementation, and provide some considerations about the performance achieved by TRIM.

\subsection{Prototype implementation}

Fig. 7 shows the testbed used to run all experiments. The elements of the TRIM architecture (the MN and the AS) have been implemented using Java and the JAIN SIP API. ${ }^{6}$ All the entities of the core IMS were deployed using the Open IMS Core implementation ${ }^{7}$ in a single virtual ma chine running the Ubuntu 9.10 Linux distribution (all the machines used in this testbed were configured with the same Linux distribution). A DNS server was also configured in that virtual machine so all equipments use the same DNS server in order to resolve the ims.net virtual domain.

\footnotetext{
${ }^{6}$ JAIN SIP Developer Tools, http://jain-sip.dev.java.net/

7 http://www.openimscore.org/.
} 


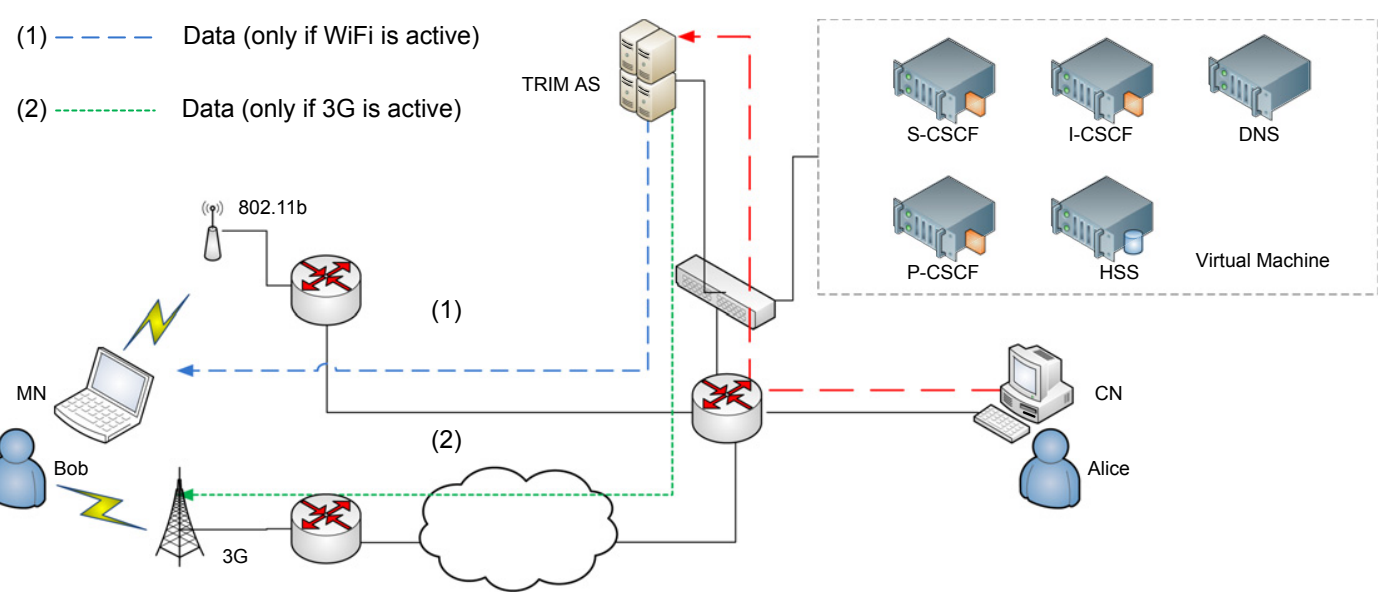

Fig. 7. Testbed.

The TRIM AS and one address translator were deployed in a single machine, which was attached to the same local area network of the core IMS (in the following, we will use the term TRIM AS for both entities). In the experiments, we have a user Alice registered at the $\mathrm{CN}$, and another user Bob regis tered at the MN. Alice and Bob exchange data using a mobil ity unaware application. While the $\mathrm{CN}$ is always attached to a wired network, the MN has two wireless interfaces: a wireless $802.11 \mathrm{~g}$ interface and a 3G/GPRS interface.

The TRIM AS is registered in the HSS of the core IMS and the users Alice and Bob have both an IP Multimedia Private Identity (IMPI) and an IP Multimedia Public Identity (IMPU) configured in the HSS and associated to the TRIM AS.

The data forwarding part of the prototype has been implemented using the NAT functionality included in $\mathrm{Li}$ nux, by means of the iptables interface. ${ }^{8}$ Whenever a hand over is performed, the corresponding addresses configured in the MN and AS must be changed, requiring dynamic changes to the Linux NAT. As we show in the following sec tions, this functionality is not implemented in the Linux NAT services. Although we have been able to make a func tional prototype of the solution using the standard Linux NAT, it is worth to notice that we would need a specific implementation of the address translator functionality of our proposal to overcome some limitations of the proto type that will be described later.

In this section, we will use the following address nomenclature:

- $I P_{C N}$ is the address used by the $\mathrm{CN}$ to connect with the TRIM AS.

- $I P_{A S C N}$ and $I P_{A S M N}$ are the addresses used by the TRIM

AS to connect with the $\mathrm{CN}$ and the MN, respectively.

- $I P_{M N} 3 G$ is the address used by the MN in the 3G leg.

- $I P_{M N}$ WLAN is the address used by the MN in the WLAN leg.

- $I P_{M N ~ L O}$ is the address used by the $\mathrm{MN}$ in the loopback interface. Note that this is the internal address available to the applications.

\footnotetext{
${ }^{8}$ See http://www.netfilter.org.
}

In the validation, UDP and TCP experiments follow the same procedure. This procedure consists of a set of steps that are described next.

The MN is registered in the IMS core by issuing a REG ISTER message containing its IP address. In these particular experiments we always started in the $3 \mathrm{G}$ leg so the IP ad dress initially registered in the IMS core is $I P_{M N} 3 G$. Once the $\mathrm{MN}$ is registered in the IMS core, it opens a communi cation with the $\mathrm{CN}$, by issuing an INVITE message which will reach the $\mathrm{CN}$ through the AS. In all cases, the applica tion running at the $\mathrm{MN}$ is listening at the address $I P_{M N}$ LO (private address manually assigned to the loopback interface). ${ }^{9}$

At this point, the $\mathrm{MN}$ and the AS have already config ured their NAT modules. In the $\mathrm{CN}$ to MN direction, the NAT configuration allows the AS to forward all the packets arriving at the AS with destination the MN to the current MN's location ( $\left.I P_{M N} 3 G\right)$. The NAT configuration at the MN allows to address the packets of the session, received at $I P_{M N 3 G}$, to the address $I P_{M N} L O$, where the application is lis tening. In the $\mathrm{MN}$ to $\mathrm{CN}$ direction, the NAT configuration at the MN allows to change the source IP address $\left(I P_{M N} L O\right)$ of the packets belonging to the session to the address of the active interface $\left(I P_{M N} 3 G\right)$. The NAT configuration in the AS allows to forward the packets received from the $M N$ to the $\mathrm{CN}$, changing the source IP address from $I P_{M N} 3 G$ to $I P_{A S C N}$, the destination IP address from $I P_{A S} M N$ to $I P_{C N}$, and the transport ports, as illustrated in Fig. 5.

After a certain time, the MN decides to perform a hand over to the WLAN interface. In order to do so, the MN reg isters itself again with the core IMS with the new IP address obtained ( $I P_{M N}$ WLAN $)$.

Once the new IP address is registered within the IMS, the MN modifies the multimedia session by issuing a re INVITE towards the AS, which is able to start sending the packets to the new MN address, by changing its NAT con figuration to reflect the IP address in use in the $\mathrm{MN}$

\footnotetext{
9 Please note that conceptually this is similar to use the loopback address 127.0.0.1, but the Linux kernel does not allow changing the destination IP address of an incoming packet to the loopback address.
} 
$\left(I P_{M N}\right.$ WLAN). In addition, the MN configures its NAT accord ing to its new IP address, and also sets up the correct de fault route through the new interface.

\subsection{UDP Setup}

In order to validate the solution under the assumption of UDP traffic, we tested the architecture by sending from the $\mathrm{CN}$ to the $\mathrm{MN}$ a video stream of variable bit rate. The experiment set up and the different steps followed to per form the handover are the ones described in Section 4.1.

Fig. 8 shows a complete cycle of handover, starting in the 3G leg and moving to the WLAN for a few seconds be fore coming back to the $3 \mathrm{G}$ interface. The solid line in Fig. 8 shows the amount of traffic received through the $3 \mathrm{G}$ inter face while the dotted line corresponds to the traffic re ceived in the WLAN interface. It is important to note that in this experiment we were not saturating the network, since our aim was just to validate the proposal. The hand over to WLAN in Fig. 8 occurs between the 66.5 and $67 \mathrm{~s}$ as can be seen in the close up of the figure. The close up also shows how packets are routed through the WLAN interface exactly at the same moment that the $3 \mathrm{G}$ interface drops to zero. As conclusion from this graph, in the case of UDP flows, our solution is able to achieve zero packet loss. In next section, where we worked with TCP traffic, we pro vide insights of how the nature of TCP traffic, the linux routing mechanisms and the NAT implementation compli cate the prototype functionality.

\subsection{TCP Setup}

This section focuses on the validation of the proposed solution when TCP traffic is used. Unlike in the UDP case, in this scenario we only validate the user plane as a proof of concept, hence the handovers were triggered manually without SIP intervention (the interaction with SIP was al ready tested in the UDP setup). In order to develop a proto type of the proposed solution we opted to use the NAT functionality of the Linux Kernel, as explained in Section 4.1.

The NAT mechanism in Linux relies on the connection tracking (conntrack) properties of the kernel. The first time a packet (matching a certain rule in the NAT table) traverse the kernel, an entry in the conntrack system is created and

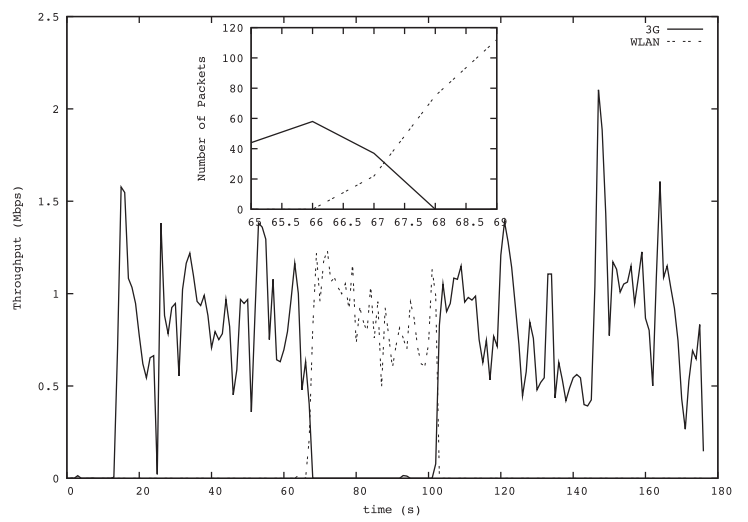

Fig. 8. Handover of UDP data flow.

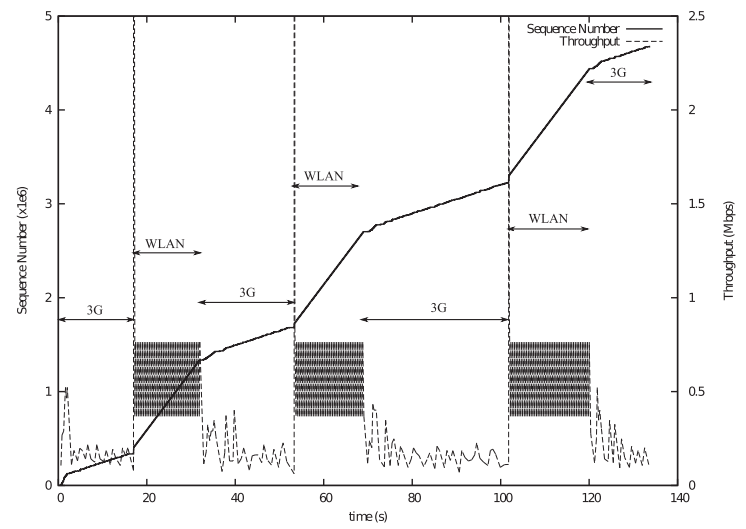

Fig. 9. Handover of TCP data flow.

no more packets of this certain flow transverse the NAT table. In order to modify the packets with the new IP addresses after a handover, we opted to remove the entry in the conntrack table each time a handover is performed. Due to TCP bidirectional nature, a conntrack entry matching the complete bidirectional flow is set, so once the conntrack entry has been removed, the reception of any TCP segment, data or ACK, prior to the setup of the new entry on the NAT table will register a new entry in the conntrack table. This leads to two workarounds in our prototype for TCP traffic: the removal of conntrack entries after the handover, and the shutdown of the old interface in the handover proce dure, so we have hard handovers in the TCP scenario.

For the validation of the solution with TCP, we used the iperf ${ }^{10}$ tool to generate the traffic from the $\mathrm{CN}$ to the $\mathrm{MN}$. We restricted the traffic generated by the iperf tool to 500 Kbps. Fig. 9 shows a Sequence number/Throughput vs Time plot with several handovers between $3 \mathrm{G}$ and WLAN following the steps mentioned in Section 4.1. If we focus on the Throughput graph we can see how the bandwidth obtained from the WLAN is clearly higher than the one obtained while using 3G. The throughput in the WLAN is limited by the appli cation rate, while in the $3 \mathrm{G}$ it is limited by the network band width. ${ }^{11}$ Note that after each handover from 3G to WLAN, there is a throughput peak caused by the sending of the pack ets buffered by TCP during the $3 \mathrm{G}$ period, which now can be delivered at a higher rate through the WLAN. The peak lasts for a short time because even if some packets are lost during the handover, the RTT is very small in the WLAN case (in the order of $2 \mathrm{~ms}$ ), enabling a very fast increase of the congestion window (note also that selective acknowledgements and fast retransmit are used in our version of TCP).

\subsection{Considerations about performance}

This section covers a set of considerations about the performance achieved by TRIM. In this proposal, an inter mediate element (an address translator located in the home network of the MN) is placed in the media path

\footnotetext{
10 http://iperf.sourceforge.net.

11 The experiments for TCP and UDP were done in different days, and the achieved throughput through the $3 G$ was different due to network conditions.
} 
between the $\mathrm{MN}$ and the $\mathrm{CN}$. The behavior of the address translator is similar to a home agent in Mobile IP, from the point of view that the data traffic has to go through the home network. Nevertheless, and although this may increase the end to end delay between the MN and the $\mathrm{CN}$, it does not prevent real time communications. As an example, conferencing in IMS, as it has been defined by 3GPP, uses an intermediate element (i.e. the Multimedia Resource Function Processor) to receive, combine and redistribute media streams to the conference participants.

Another parameter to characterize the performance achieved by a mobility management solution is the hand over delay. To estimate the handover delay achieved by TRIM, we assume that a MN is participating in a multimedia session with a $\mathrm{CN}$ via a given access network. In this situa tion, we define the handover delay, $T_{h o}$, as the time that elapses from the instant the UE moves to the new network until it starts receiving data through this new network. To estimate $T_{h o}$, we identify all the delays involved in the hand over process:

- $T_{\text {attach }}$ is the time necessary to attach the MN to the new network.

- $T_{\text {act-sig }}$ is the time necessary to activate a signaling channel in the new network (e.g. a primary PDP context in the case of UMTS). This delay includes the time to configure the MN with a new IP address, and to discover the address of the new P CSCF (if a P CSCF in the new network is to be used).

- $T_{\text {register }}$ gathers the delay corresponding to the IMS reg istration of the MN to the S CSCF.

- $T_{\text {sip }}$ gathers the delay corresponding to the INVITE trans action through the new network. This is the time that elapses from the instant the MN generates the INVITE request until it receives the corresponding OK response (after sending this response, the address translator starts forwarding the media belonging to the session to the new location of the MN). $T_{s i p}$ also includes the time necessary to execute the resource reservation pro cedures in the new network (i.e. the time necessary to activate the transport bearers that are necessary to deli ver the media).

Taking this delays into consideration, the handover delay can be estimated as: $T_{\text {ho }}=T_{\text {attach }}+T_{\text {act-sig }}+T_{\text {register }}+T_{\text {sip }}$.

It is important to highlight that this handover delay is the same that can be achieved with the IMS service conti nuity procedures defined by 3GPP [7], as these procedures comprise attaching and gaining IP connectivity to the new network, registering and initiating a new multimedia ses sion towards a Service Centralization and Continuity $\mathrm{AS}^{12}$ (SCC AS).

TRIM can achieve zero packet loss during soft hand overs (see Section 4.2). However, hard handovers may af

\footnotetext{
${ }^{12}$ We do not consider here the delay of the signaling process executed between the SCC AS and the $\mathrm{CN}$ to update the session over the remote access, as this process could run in parallel to the session setup between the MN and the SCC AS. However, note that this signaling is not required in TRIM, as the $\mathrm{CN}$ always observes stable remote addressing information for the MN.
}

fect performance because, during the handover delay, we cannot continue using the old network while setting up the communication through the new one. It is necessary to develop optimizations to TRIM to achieve zero packet loss in hard handover scenarios. We plan to address this is sue as a future work (see conclusions).

\section{Related work}

Providing mobility support in IP networks is a well known problem. At the IP layer, the Mobile IP protocols ([2] for IPv6, and [1] for IPv4) have been developed to pro vide mobility support. An alternative solution for SIP based services over IP is to provide the mobility by means of SIP signaling [6]. In a SIP based mobility solution, the mobility is not transparent for the application that has to deal with the change of address when one node moves. This has also an impact on the $\mathrm{CN}$ which is aware and has to do operations when the MN moves. A SIP based mobility solution has the advantage of not depending on having mobility functional ity at the IP layer. This is specially important in IMS based networks, because the integration of MIP in the IMS frame work is complex and requires modifications in IMS.

In the following subsections we provide an overview of related works, differentiating between plain SIP mobility and IMS based mobility.

\subsection{Mobility with plain SIP}

In recent years there has been several works studying terminal mobility using SIP such as the seminal work in [13], performance studies like [14], support of mobility across heterogeneous domains [15], and the use of Mobile IPv6 and SIP in heterogeneous networks [16].

The works in [17] and [18] are very related to our pro posal. The authors propose to use an intermediate node to hide the mobility of the MN to the CN. The SBC could be a B2BUA (Back to back User Agent) or a special SIP proxy so both the $\mathrm{MN}$ and the $\mathrm{CN}$ exchange signaling through this intermediate entity. All media flows pass through the intermediate node, again to hide mobility to the $\mathrm{CN}$. Never theless, in this proposal the mobility is not transparent for the application in the MN, which has to deal with the change of address when moving.

\subsection{Mobility in IMS based networks}

Faccin et al. [19] is one of the firsts works tackling the problem of integrating MIP in IMS. This work covers sev eral scenarios combining IMS in 3GPP and IMS in 3GPP2 with MIPv4 and MIPv6, but there are several challenges in order to integrate MIP in IMS as will be discussed next.

The addressing considerations when using MIP with SIP are discussed in [19] with an analysis of the usage of the $\mathrm{HoA}^{13}$ or the $\mathrm{CoA}^{14}$ at the MN. The HoA should be used both for SIP signaling and for session establishment (data plane)

\footnotetext{
${ }^{13}$ Home Address, permanent address used by the MN and topologically valid in its home network.

${ }^{14}$ Care-of Address, temporal address used by the MN while visiting a network and topologically valid in that network.
} 
to obtain the advantages of transparent mobility that MIP provides. The CoA would be transparent to the application layer (both for SIP and the application) and MIP would take care, at the IP layer, of the matching between the CoA used to send and receive traffic in the $\mathrm{MN}$ and the HoA. Unfortu nately, in IMS we cannot simply use the HoA, because re sources are reserved in the access network for the traffic corresponding to the SIP service. Because packets coming to the MN have to have the CoA as the destination IP ad dress, and packets coming from the MN have to have the $\mathrm{CoA}$ as source address, in both cases with the HoA present in a special extension header (IPv6) or in an inner IP header (when a tunnel is used in IPv4 or in IPv6) the P CSCF needs to know the CoA as well as the HoA in order to install the proper filters in the network edge, so it is not enough that SIP manages only the HoA. In [19] authors argue that with MIP for IPv6 (MIPv6), the MN could use MIPv6 signaling (a Binding Update) to inform the P CSCF of the association CoA HoA. This is true at the IP layer, but we would still have the problem that SIP would be unaware of the CoA. This problem was already studied in [3] for cdma2000 and WLAN access, and in [4,5] for GPRS/UMTS access. They basically propose to have fully MIP aware P CSCFs and access routers introducing an interface between the MIP binding tables and the proper functionalities, but this means introducing changes in the IMS specifications.

Related with the previous item, there is also a problem with the registration process as, when using MIP, the MN has to register the HoA. The P CSCF will reject the registra tion because the HoA is not a valid IP address inside its ac cess network domain.

Faccin et al. [19] also deal with the return routability (RR) test when using MIPv6. In RR, the CN challenges the $\mathrm{MN}$ to verify its reachability using the CoA. In order to do so, the $\mathrm{CN}$ sends challenges to the CoA and HoA so the $\mathrm{MN}$ receives these two messages and sends a response using both challenges. This test is performed at a certain frequency (a few minutes) so there is an extra overhead in the communication between the MN and the P CSCF in case the $\mathrm{P}$ CSCF behaves like a $\mathrm{CN}$ as proposed by the authors.

In [20], the authors propose supporting mobility for IMS based IPTV peer to peer services by combining MIP with IMS, and using a context transfer mechanism to sup port efficient handovers. This proposal requires modifica tions to the IMS infrastructure to support the integration of MIP.

Another approach to handle mobility has been pro posed by 3GPP in [7]. This specification describes a service that supports the use of session transfer mechanisms to maintain service continuity in the event of terminal mobil ity, for the case when this mobility is not transparent to the IMS session. In this solution, session transfer procedures are initiated by the MN and are handled by a Service Cen tralization and Continuity Application Server (SCC AS), which fits in the signaling path between the MN and the $\mathrm{CN}$. After obtaining IP connectivity in a new network, the MN can preserve the continuity of an active multimedia session by starting the session transfer procedures. Specif ically, it registers to the S CSCF from the new network and it initiates a new multimedia session towards the SCC AS by sending an INVITE request. The MN includes in this re quest an SDP payload, with its current addressing informa tion in the new network. Upon receiving the INVITE request, the SCC AS matches the new multimedia session with the session to be transferred, and modifies the remote leg of the session providing the CN with the SDP payload indicated by the MN. This way, the new location of the $\mathrm{MN}$ is available to the $\mathrm{CN}$ and session continuity can be maintained.

Nevertheless, mobility management procedures de scribed in this technical specification are not transparent to applications at the MN and the CN. Specifically, when the $\mathrm{MN}$ moves to a new network, its local applications must generate a new SDP description and trigger a new session setup towards the SCC AS. Applications running at the $\mathrm{CN}$ must be contacted to update the addressing information corresponding to the MN. In addition, any net work socket bound to the previous IP address of the MN becomes invalid. Therefore, applications at the MN and $\mathrm{CN}$ may need to re configure or to open new network sock ets. This is particularly problematic in the case of TCP transport, as active TCP connections need to be re established.

Compared with the related work above, our proposal has the advantage of not requiring a MIP deployment, therefore avoiding the problems of integrating MIP in IMS based networks, while keeping mobility transparent to the applications. In addition, it does not require any modifications to the IMS infrastructure. In the home net work, the proposal introduces a SIP Application Server (i.e. the TRIM AS) and an address translator. In the UE, the proposal only requires some new functions at the $\mathrm{MN}$, but those can be installed as a simple software upgrade.

\section{Conclusion}

This paper proposes TRIM, an architecture to provide mobility support in IMS based networks. TRIM is based on SIP signaling, but unlike other approaches to offer mobility support in IP networks based on SIP, TRIM makes mobility transparent to applications, which do not have to do any operation to support a change of access network by the mobile node. This functionality is similar to the one provided by Mobile IP, but the compatibility of Mobile IP and IMS requires modifications to the IMS specifications, a requirement that TRIM does not have. We have tested a prototype of TRIM in a testbed combining 3G and IEEE 802.11 access technologies, and an IMS core. In the exper iments we showed that TRIM correctly supports mobility both for UDP and TCP user traffic.

There are several lines of future research for TRIM. TRIM forwards the user traffic through a network element in the mobile node home network, which is similar to Mobile IP without route optimization. We want to explore an optimi zation to place this network element within or close to the network being visited by the mobile node. Additionally we want to study the performance of handovers when only one network interface is available in the mobile node and soft handovers are not possible. An approach to improve 
performance could be to develop optimizations based on buffering in the intermediate network element.

\section{Acknowledgements}

This article has been partially granted by the European Community Seventh Framework Programme (FP7/2007 2013) under grant agreement 214994 (CARMEN project), and by the Madrid Community through the MEDIANET project (S 2009/TIC 1468).

\section{References}

[1] C. Perkins, IP Mobility Support for IPv4, RFC 3344, Internet Engineering Task Force, August 2002

[2] D. Johnson, C. Perkins, J. Arkko, Mobility Support in IPv6, RFC 3775, Internet Engineering Task Force, June 2004.

[3] T. Chiba, H. Yokota, A. Dutta, D. Chee, H. Schulzrinne, Performance analysis of next generation mobility protocols for IMS/MMD networks, in: Wireless Communications and Mobile Computing Conference, 2008. IWCMC'08. International, IEEE, 2008, pp. 68-73.

[4] T. Renier, K. Larsen, G. Castro, H. Schwefel, Mid-session macromobility in IMS-based networks, IEEE Vehicular Technology Magazine 2 (1) (2007) 20-27.

[5] X. Chen, J. Wiljakka, Problem statements for MIPv6 interactions with GPRS/UMTS packet filtering, Internet draft, Internet Engineering Task Force (2007).

[6] H. Schulzrinne, E. Wedlund, Application-layer mobility using SIP, ACM SIGMOBILE Mobile Computing and Communications Review 4 (3) (2000) 57.

[7] 3GPP, IP Multimedia Subsystem (IMS) service continuity: Stage 2, TS 23.237 v9.6.0 Release 9, 3rd Generation Partnership Project (3GPP), September 2010.

[8] 3GPP, IP Multimedia Subsystem (IMS); Stage 2, TS 23.228, 3rd Generation Partnership Project (3GPP), March 2010.

[9] J. Rosenberg, H. Schulzrinne, G. Camarillo, A. Johnston, J. Peterson, R. Sparks, M. Handley, E. Schooler, SIP: Session Initiation Protocol, RFC 3261, Internet Engineering Task Force, June 2002.

[10] M. Handley, V. Jacobson, C. Perkins, SDP: Session Description Protocol, RFC 4566, Internet Engineering Task Force, July 2006.

[11] 3GPP, Internet Protocol (IP) multimedia call control protocol based on Session Initiation Protocol (SIP) and Session Description Protocol (SDP); Stage 3, TS 24.229 v9.3.1 Release 9, 3rd Generation Partnership Project (3GPP), September 2010.

[12] F. Brockners, S. Bhandari, V. Singh, V. Fajardo, Diameter network address and port translation control application, Internet draft, IETF, 2010.

[13] E. Wedlund, H. Schulzrinne, Mobility support using SIP, in: Proceedings of the Second ACM International Workshop on Wireless Mobile Multimedia, ACM, 1999, pp. 76-82.

[14] N. Nakajima, A. Dutta, S. Das, H. Schulzrinne, Handoff delay analysis and measurement for SIP based mobility in IPv6, in: IEEE International Conference on Communications, 2003. ICC'03, vol. 2, IEEE, 2003, pp. 1085-1089.

[15] M. Cardenete-Suriol, J. Mangues-Bafalluy, M. Portoles-Comeras, M Requena-Esteso, M. Gorricho, VoIP performance in SIP-based vertical handovers between WLAN and GPRS/UMTS networks, in: Communications, 2007, in: IEEE International Conference on ICC'07, IEEE, 2007, pp. 1973-1978.

[16] M. Bernaschi, F. Cacace, G. Iannello, M. Vellucci, Mobility management for VoIP on heterogeneous networks: evaluation of adaptive schemes, IEEE Transactions on Mobile Computing (2007) 1035-1047.

[17] S. Salsano, L. Veltri, A. Polidoro, A. Ordine, Architecture and testbed implementation of vertical handovers based on SIP session border controllers, Wireless Personal Communications 43 (3) (2007) 10191034.

[18] E. Boysen, H. Kjuus, T. Maseng, Proactive handover in heterogeneous networks using SIPs, in: Seventh International Conference on Networking, IEEE, 2008, pp. 719-724.

[19] S. Faccin, P. Lalwaney, B. Patil, IP multimedia services: analysis of mobile IP and SIP interactions in 3G networks, Communications Magazine, IEEE 42 (1) (2005) 113-120.

[20] I. Vidal, J. Garcia-Reinoso, A. de sla Oliva, A. Bikfalvi, I. Soto, Supporting mobility in an IMS-based P2P IPTV service: a proactive context transfer mechanism, Computer Communications 33 (14) (2010) 1736-1751.

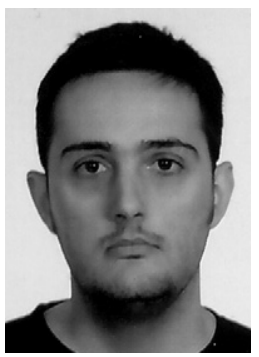

Dr. Ivan Vidal received the Telecommunication Engineering degree in 2001 from the University of Vigo, and the Ph.D. in Telematics Engineering in 2008 from the University Carlos III of Madrid. His research interests include network security, multiparty services based on network multicast in the IP Multimedia Subsystem (IMS) and peer-to-peer overlays for multimedia streaming in the Internet and IMS-based next generation networks.

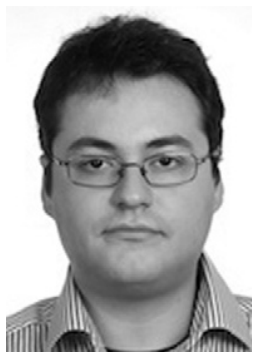

Antonio de la Oliva received his Telecommunications Engineering degree in the Universidad Carlos III de Madrid in 2005 and his Ph.D. in Telematics by the same university in 2008. He is currently working as associate professor at Universidad Carlos III de Madrid. He is participating in FP7 CARMEN project and his research topics are wireless mesh, heterogeneous networks and IEEE 802.21 where he has performed several contributions to the standard.

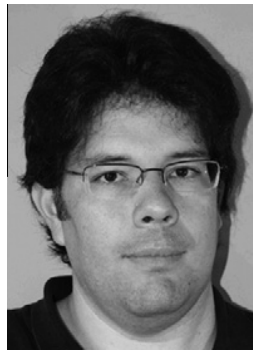

Jaime Garcia received the Telecommunications Engineering degree in 2000 from the University of Vigo, Spain and the Ph.D. in Telecommunications in 2003 from the University Carlos III of Madrid, Spain. He is currently an associate professor at Univ. Carlos III of Madrid having joined in 2002 and he has published over 35 papers in the field of broadband computer networks in magazines and congresses. He has been involved in several international and national projects related with protocol design, user localization, broadband access, peer-to-peer overlays, Next Generation Networks and signaling protocols like the EU IST MUSE, the EU NoE CONTENT, the Spanish BioGridNet and currently he is working in the Spanish MEDIANET project related with video streaming distribution over peer-to-peer networks in the future Internet.

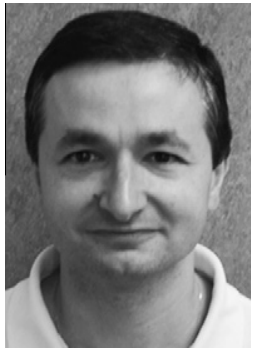

Ignacio Soto received a Telecommunication Engineering degree in 1993, and a Ph.D. in Telecommunications in 2000, both from the University of Vigo, Spain. He was a research and teaching assistant in Telematics Engineering at University of Valladolid from 1993 to 1999. In 1999 he joined University Carlos III de Madrid, where he was an associate professor since 2001 until 2010. In 2010 he joined Universidad Politcnica de Madrid as associate professor. His research activities focus on mobility support in packet networks and heterogeneous wireless access networks. He has been involved in international and national research projects related with these topics, including the EU IST Moby Dick and the EU IST Daidalos projects. He has published several papers in technical books, magazines and conferences, lately in the areas of efficient handover support in IP networks with wireless access, network mobility support, and security in mobility solutions. 